\title{
Feature Extraction of Performance Variables in Elite Half-Pipe Snowboarding Using Body Mounted Inertial Sensors
}

\author{
J. W. Harding ${ }^{1,2,3,4}$, J. W. Small ${ }^{3}$, D. A. James ${ }^{3,5}$ \\ ${ }^{1}$ Department of Physiology, Australian Institute of Sport, Australia \\ ${ }^{2}$ Applied Research Centre, Australian Institute of Sport, Australia \\ ${ }^{3}$ Centre for Wireless Monitoring and Applications, Griffith University, Australia \\ ${ }^{4}$ Olympic Winter Institute of Australia, Australia \\ ${ }^{5}$ Centre of Excellence for Applied Sport Science Research, Queensland Academy of Sport, Australia
}

\begin{abstract}
Recent analysis of elite-level half-pipe snowboard competition has revealed a number of sport specific key performance variables (KPV's) that correlate well to score ${ }^{1}$. Information on these variables is difficult to acquire and analyse, relying on collection and labour intensive manual post processing of video data. This paper presents the use of inertial sensors as a user-friendly alternative and subsequently implements signal processing routines to ultimately provide automated, sport specific feedback to coaches and athletes. The author has recently shown that the key performance variables (KPV's) of total air-time (TAT) and average degree of rotation (ADR) achieved during elite half-pipe snowboarding competition show strong correlation with an athlete's subjectively judged score ${ }^{1}$. Utilising Micro-Electrochemical System (MEMS) sensors (tri-axial accelerometers) this paper demonstrates that air-time (AT) achieved during half-pipe snowboarding can be detected and calculated accurately using basic signal processing techniques. Characterisation of the variations in aerial acrobatic manoeuvres and the associated calculation of exact degree of rotation (DR) achieved is a likely extension of this research. The technique developed used a two-pass method to detect locations of half-pipe snowboard runs using power density in the frequency domain and subsequently utilises a threshold based search algorithm in the time domain to calculate air-times associated with individual aerial acrobatic manoeuvres. This technique correctly identified the air-times of 100 percent of aerial acrobatic manoeuvres within each half-pipe snowboarding run $\left(\mathrm{n}=92\right.$ aerial acrobatic manoeuvres from 4 subjects) and displayed a very strong ${ }^{12}$ correlation with a video based reference standard for air-time calculation $(\mathrm{r}=0.78 \pm 0.08$; $\mathrm{p}$ value $<0.0001$; $\mathrm{SEE}=0.08 \times / \div 1.16$; mean bias $=-0.03 \pm 0.02 \mathrm{~s})$ (value \pm or $\times / \div 95 \% \mathrm{CL})$.
\end{abstract}

Keywords: Snowboarding, Half-pipe, Inertial Sensors, Performance Assessment, Accelerometers, Gyroscopes, Snowboard Competition, Judging.

\section{INTRODUCTION}

\subsection{Elite half-pipe snowboarding}

Half-pipe snowboarding is an aerial acrobatic routine performed by snowboard athletes on a half-pipe constructed of snow. Although the dimensions of snowboard half-pipes vary within different mountain resorts, Olympic and World Cup standard half-pipes are generally $160 \mathrm{~m}$ in length, $18 \mathrm{~m}$ in width, $6.0 \mathrm{~m}$ deep and are situated on a gradient of approximately 18 degrees (Figure 1). The objective in recreational and competitive half-pipe snowboarding is to perform well executed and stylish routines consisting of complex aerial acrobatic manoeuvres that are executed as high above the half-pipe lip as possible (Figure 2). Performances within a competition environment are currently assessed via subjective measures from a panel of judges. Previous research using labour intensive video based methods has however found a very large $\mathrm{e}^{12}$ correlation between the combined performance variables of total air-time (TAT) and average degree of rotation (ADR) achieved during elite half-pipe snowboarding competition and an athlete's subjectively judged score ${ }^{1}$. This paper has focussed primarily on providing sport scientists and elite level coaches the capacity to routinely monitor half-pipe snowboarding performance in the field using micro technology sensors, now emerging in routine use in sports science applications. 


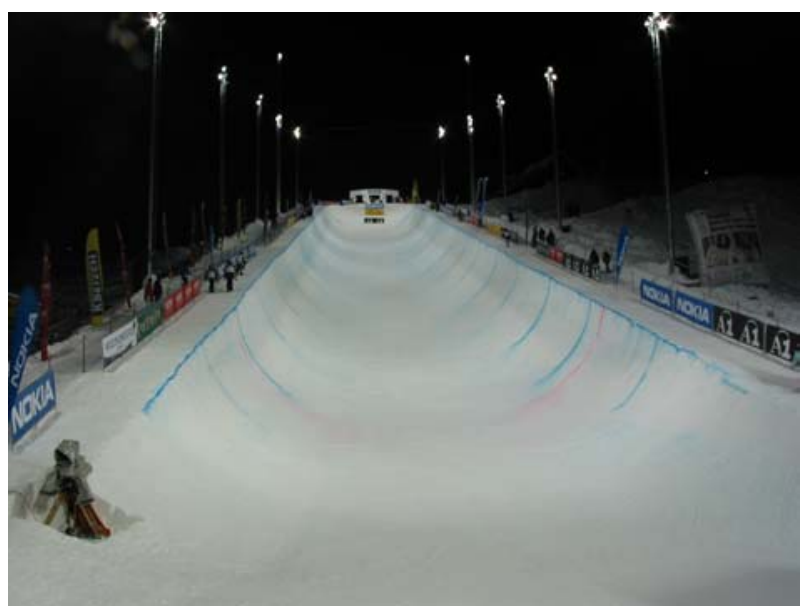

Figure 1. World Cup standard snowboard half-pipe. Kreischberg, Austria 2006.

\subsection{Monitoring of sports performance in the field}

Laboratory based performance assessment of half-pipe snowboarding is currently impossible. Subsequently, there have been a number of recent attempts to develop or use current technological based systems to quantify sport specific variables associated with snowboarding in the field. For example the kinematics and kinetics associated with snowboarding turns on-snow have been quantified using an electromagnetic tracking system (FASTRAK, Polhemus) ${ }^{2}$. Using this electromagnetic tracking system, objective information pertaining to angular displacement and joint moment torque information related to snowboard turns can be quantified upon post processing of data captured on-snow ${ }^{2}$. The same electromagnetic tracking system was also utilised by another group of researchers who were able to provide quantitative data on the degree of dorsiflexion, eversion and external rotation of the ankle joint complex during on-snow trials of two types of snowboard boots ${ }^{3}$. By utilising the ability to assess quantitative data captured in the field it was shown that 'step-in' snowboard boots (which are stiffer than regular snowboard boots) allow less dorsiflexion, eversion and external rotation than softer boots, thereby providing quantitative evidence as to why 'step-in' boots are associated with a lower rate of fractures of the talus than softer boots ${ }^{3}$. The development and associated integration of a dynamometric platform focussed upon field based load data acquisition in snowboarding has also been presented ${ }^{4}$. This dynamometric device is able to measure all load components transmitted between the boots and snowboard bindings without interfering with current snowboard binding technology. This platform has the capacity to provide quantitative information on the forces and torques (in 10 directions) imparted by the snowboard boot onto the bindings. Field tests utilising this device showed that positive forces are witnessed at the front of the binding plate during a front-side turn and negative forces are witnessed at the front of the bindings in a back side turn ${ }^{4}$. It is believed elite-level half-pipe snowboarding is well positioned to take advantage of this newly discovered potential to quantitatively measure sport specific variables in the field and the associated shift in performance assessment ideology.

\subsection{Micro-technology's potential within field based performance monitoring}

By taking advantages of the advancements in microelectronics and other micro technologies it is now possible to build instrumentation that is small and unobtrusive enough for a number of field based sporting and clinical applications. The capacity of tri-axial accelerometers and tri-axial rate gyroscopes to measure human motion thousands of times per second in multiple axes and at multiple points on the body is well established and the capacity for on-board data storage negates the requirement of equipment tethering ${ }^{6}$. The information on the kinematics of technical skill based sports (such as snowboarding) that were generated by the electromagnetic tracking system ${ }^{2}$ as they occurred in the natural athletic environment was a worthwhile goal and exemplifies current field based performance assessment ideologies. The bulky electromagnetic tracking system worn by the subject and the ten kilogram measurement system carried by the researcher tethered to the subject ${ }^{2}$ however, is far from the unobtrusive measurement systems this shift in ideology is targeting. It is 
hypothesised that the weight of this monitoring system acting on the athlete alone would constrict and alter their normal movement patterns removing any relevance to real life snowboarding technique. A monitoring system such as this (bulky construction and tethered requirements) would most likely prevent any data collection from elite-level athletes training or competing in aerial acrobatic events with elements of risk involved, such as half-pipe snowboarding. The capacities afforded by micro-technologies such as tri-axial accelerometers, tri-axial rate gyroscopes and tri-axial magnetometers possess enormous potential in providing similar information without the problems associated with size, weight and the necessity for tethering. As an example, researchers have used a combination of small, lightweight force sensing resistors $\left(\mathrm{ADXL} 210 \mathrm{E} \pm 10 \mathrm{~g}\right.$ accelerometers and ADXRS300 $\pm 300 \mathrm{~d} . \mathrm{s}^{-1}$ rate gyroscopes) to collect measurements under skier's feet and thereby gain an insight into the ground reaction forces and an athlete's weight balance in the field ${ }^{7}$. By combining this technology with video analysis software (SKI) they provided visual feedback of a skier's movement patterns in parallel to quantitative data from the accelerometers and rate gyroscopes ${ }^{7}$. The absence of weight, size and tether in the monitoring device allows the athlete to move freely and perform just as they would when not under scientific scrutiny. Although accuracy and reliability of measurements are extremely important aspects of technology based in-field monitoring, the benefit of such scientific rigour is lost if athletes alter their performance and movement patterns as a result of scientific scrutiny. Furthermore, the author believes it is imperative that the field-based monitoring techniques now afforded by micro-technologies are focussed upon relevant and practical benefit to those who are being monitored.

\subsection{In-field monitoring with a practical purpose}

The aim of this relatively new area of athlete monitoring should not be to instrument everything on the human body and sporting equipment but rather to understand what information is required to enhance performance and to elucidate the most appropriate sensors to provide such information ${ }^{6}$. The interpretation of this information into something suitable for use by coaches and athletes should additionally constitute a large component of any research focus. The research that developed and utilised a dynamometric platform stated that knowledge of load histories in snowboarding will allow a deeper insight into rider's motion dynamics during carves or jumps ${ }^{4}$. Field tests utilising this device showed that positive forces are witnessed at the front of the binding plate during a front-side turn and negative forces are witnessed at the front of the bindings in a back side turn ${ }^{4}$. The ability to measure forces accurately and reliably are academically sound and do provide quantitative insight into a rider's motion dynamics during carves or jumps however, the declaration that this information will help coaches and athletes in functional evaluation and training monitoring seems to be an optimistic statement. The conclusions that force is generated on the front of the binding during a front-side turn and that force is not generated on the front of the binding during a back-side turn (it is shifted to the heel of the binding) ${ }^{4}$ is common knowledge to all those who snowboard, and as such seems to offer relatively limited insight to elite-level performance diagnostics and competition preparation. It is believed that the development and validation of this novel technological monitoring device, has been conducted with the utmost scientific rigour and has the potential to generate invaluable information of relevance to elite-level coaches and athletes. The research and line of enquiry however, seems to have occurred independently of key stakeholders and there is no evidence of the initiation or maintenance of a critical partnership with elite-level coaches and athletes required for successful integration of this concept. In terms of the focus of this paper, the author has ensured field based performance assessment and the subsequent development and integration of a technological monitoring device has been initiated with the potential benefit to elite-level coaches and athletes as a focal outcome. In the case of the preparation of elite-level athletes and the progression of performance toward competitive achievements, the research outcomes of this paper are focused upon the sport specific key performance variables (KPV's) associated with elite-level half-pipe snowboard competition success.

\subsection{Key performance variables in elite half-pipe snowboard competition}

In terms of objective information the two most important performance variables in elite-level half-pipe snowboarding competition are air-time (AT) and degree of rotation (DR). When total air-time (TAT) and average degree of rotation (ADR) are combined (multiple regression) they have been shown to exhibit a very large ${ }^{12}$ correlation with an athlete's subjectively judged score $(\mathrm{r}=0.70 ; \mathrm{p}$ value $<0.0001 ; \mathrm{n}=30$ runs $)$, and account for approximately $50 \%$ of the shared variance associated with the overall score $\left(\mathrm{r}^{2}=0.49, \mathrm{SEE}=3.97\right)$ during World Cup half-pipe snowboard competition finals $(n=2)$ in Bardonecchia Italy ${ }^{1}$. It is important to note however that this relationship is based upon two World Cup half-pipe snowboard finals and the contribution of these key performance variables toward subjectively judged scores 
can change as a result of varying competition performance levels, varying snowboard half-pipe dimensions and different judging panels. Even so the overall trend remains; that athletes with higher TAT and higher ADR, provided runs are well executed and completed cleanly, almost always perform better in terms of competition outcomes. This knowledge may enhance the capacity of coaches to monitor athlete performance progression and to set specific competition goals based upon objective information, as opposed to the current reliance on purely subjective performance indicators. Accurate and reliable quantification of these sport specific key performance variables (KPV's) immediately post run may also provide the capacity to assist current subjective judging protocols in reliably assessing competition performance ${ }^{1,8,11}$.

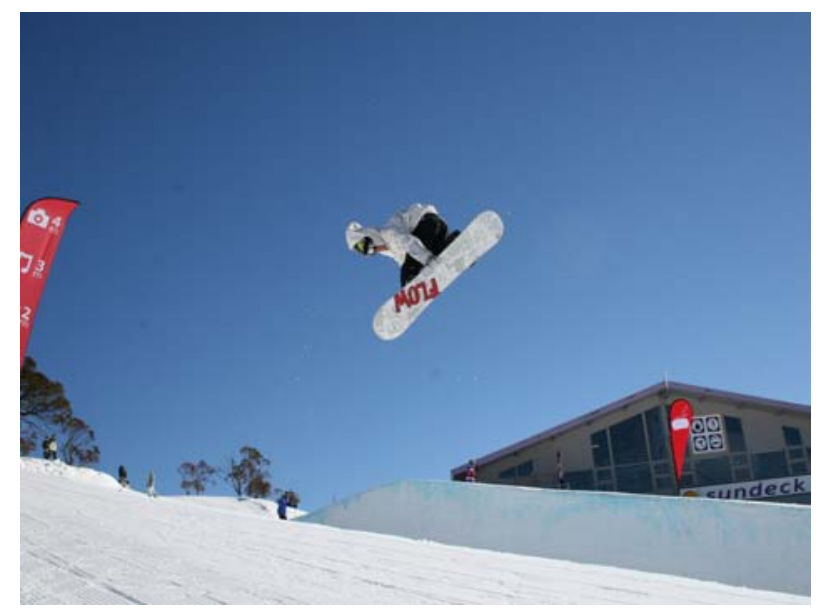

Figure 2. Australian snowboard athlete performing an aerial acrobatic manoeuvre (front-side 720 melon). AT and DR are the two most important variables in half-pipe snowboarding competition ${ }^{1,8}$. Perisher Blue Ski Resort Australia 2006.

\subsection{Automating objective feedback for elite half-pipe snowboarding}

The only currently available method for sports scientists, coaches and competition judges to calculate half-pipe snowboarding KPV's is by video based analysis and this is currently not a regular practice. Video based analysis was, and still is primarily utilised for subjective assessment of style and run execution in snowboarding. Although video may afford the capacity to calculate this objective information, the dilemma with video based analysis has always been the labour intensive nature and associated time-delay in information feedback ${ }^{1}$. The capacities afforded by microtechnologies such as tri-axial accelerometers and tri-axial rate gyroscopes to calculate KPV information associated with half-pipe snowboarding has until now, been unknown and as such unutilised. Tri-axial accelerometers in particular display very distinct raw data traces during half-pipe snowboarding and have been previously shown by the author to possess the capacity for accurate and reliable quantification of air-time that occurs during this sports discipline ${ }^{1,8,11}$. Triaxial accelerometers are easy to attach to an athlete and do not limit normal movement patterns and thus, currently seem to warrant the only realistic method of KPV analysis in half-pipe snowboarding. The capacity of micro-technology to provide sport specific KPV information automatically and immediately post run will allow coaches and competition judges to automatically and objectively assess athletic performance in the environment with which half-pipe snowboarding takes place. This outcome is mutual to the current and relatively recent shift in performance assessment and athlete monitoring ideology; moving collection and analysis away from the laboratory and into the field. This paper presents the signal processing method utilised to calculate the KPV of air-time in half-pipe snowboarding and the results of a validation study focussed upon the accuracy and the reliability of this method.

\section{METHODS}

\subsection{Subjects}

Four members of the Australian Half-pipe snowboarding team were recruited to participate in this study. Their physical characteristics for age, height, weight and $\sum 7$ skin folds were $22.18 \pm 6.93 \mathrm{yrs}, 1.7 \pm 7.8 \mathrm{~m}, 74.3 \pm 15.5 \mathrm{~kg}, 57.8 \pm 25.2$ 
$\mathrm{mm}$ (mean $\pm \mathrm{SD}$ ) respectively. Data collection was performed during the 2006 southern hemisphere winter season at Perisher Blue Ski Resort (altitude $1720 \mathrm{~m}$ ) on the resort's custom snowboard half-pipe (length $80 \mathrm{~m}$, width $18 \mathrm{~m}$, transition height $5 \mathrm{~m}$, gradient approximately 15 degrees). Experimental procedures were approved by the Ethics Committee of the Australian Institute of Sport on the $18^{\text {th }}$ August 2005 (approval number 20050808).

\subsection{Equipment and experimental procedure}

Implementation of previously developed sensors ${ }^{5,6,9}$ comprising of one tri-axial accelerometer and one tri-axial rate gyroscope were used throughout the data collection process. This paper will focus on the tri-axial accelerometer data resultant of the fact that the air-time associated with aerial acrobatic manoeuvres only relies on accelerometer data for calculation. Raw tri-axial accelerometer data was stored on board the sensor unit (256MB Trans Flash) for the duration of the data collection process and sampled post collection by a computer software suite developed in house. All accelerometer units underwent a static calibration in three axes (up/down, forward/back and left/right) prior to each data collection session aligning each axes of sensitivity with and against the direction of gravity. Analysis and development of signal processing techniques were carried out with custom software developed using Matlab ${ }^{10}$. High speed video footage of each half-pipe snowboard run was collected using a Phantom $200 \mathrm{~Hz}$ high speed video camera (VISION) from the bottom and centre of the half-pipe (Figure 3$)^{11}$. High speed video footage was collected on only one aerial acrobatic manoeuvre throughout each run as the cameras used did not have the capacity to follow a rider's progress through the half-pipe. Panning video footage of each half-pipe snowboard run was collected using a Sony $3 \mathrm{CCD} 50 \mathrm{~Hz}$ digital video camera (DVC) from the bottom and centre of the half-pipe and included every aerial acrobatic manoeuvre attempted $(\mathrm{n}=$ 92) throughout each run (Figure 3). High speed and digital video footage was analysed by video analysis software.

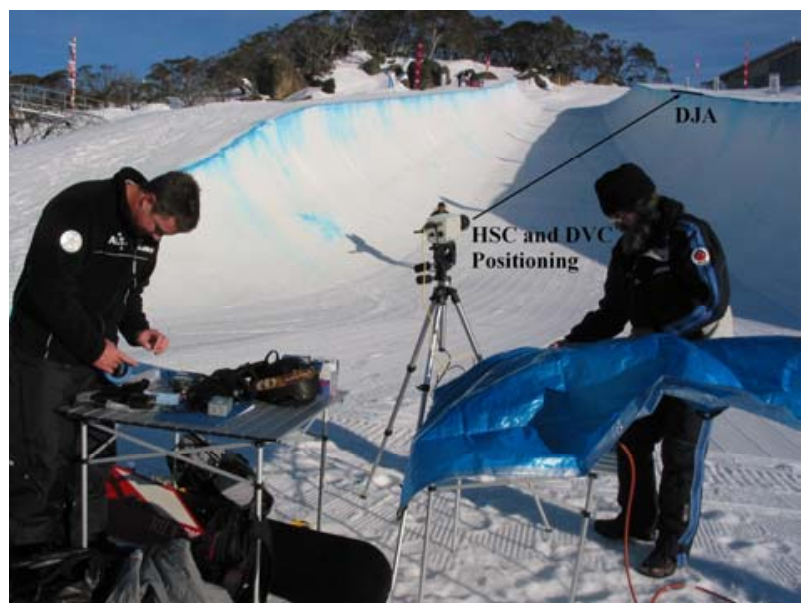

(A)

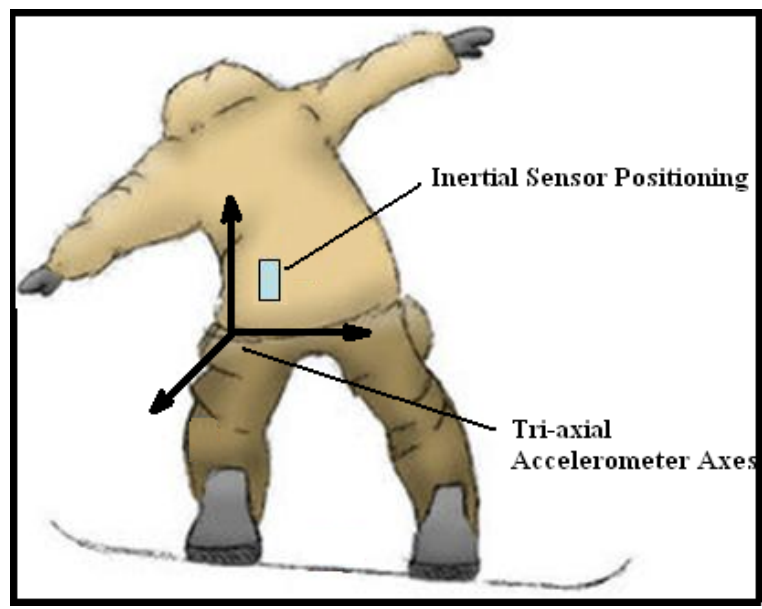

(B)

Figure 3. (A) Positioning of high speed camera, digital camera, and designated jump area during data collection ${ }^{11}$. (B) Positioning of tri-axial accelerometer and associated global coordinate axes. Perisher Blue Ski Resort Australia 2006.

\subsection{Data collection}

A sensor was attached to the lower back of each athlete, situated approximately $5 \mathrm{~cm}$ to the left of the spine. Data was collected from one individual athlete at a time during four routine Australian national team training sessions. Athletes were instructed to participate in training sessions as per normal routine, with no set number of runs or aerial acrobatic manoeuvres and no time limit imparted on data collection session. The only stipulation was that each athlete must designate a specific area of the half-pipe where they would regularly perform aerial acrobatic manoeuvres during their training. This section of the half-pipe (approximately $10 \mathrm{~m}$ in width) was termed the designated jump area (DJA) and allowed a fixed high speed camera to collect data on manoeuvres attempted over that area (Figure 3$)^{11}$. The protocol allowed athletes to train in routine fashion and eliminated any constriction or alteration of their performance. As athletes underwent their training session a panning digital video camera filmed complete half-pipe runs, a fixed high speed 
camera collected data from manoeuvres attempted over the DJA and tri-axial accelerometer data was collected for the duration of the training session. Data on 92 aerial acrobatic manoeuvres was collected during the project.

\subsection{Key performance variable definitions}

Air-time (AT) is measured in seconds and reflects the amount of time the athlete spends in the air during a half-pipe snowboarding routine. Air-time begins the first moment there is no longer contact between the snowboard and the snow (Figure 4$)^{11}$ and ends the moment any part of the snowboard comes in contact with the snow following an attempted aerial acrobatic manoeuvre. Total air-time (TAT) is measured in seconds and calculated by adding together all recorded air-times (typically 6 - 8) during a half-pipe snowboard run. Average air-time (AAT) is also measured in seconds and is calculated by dividing total air-time (TAT) by number of by the total number of aerial acrobatic manoeuvres completed throughout the duration of a half-pipe snowboarding run.

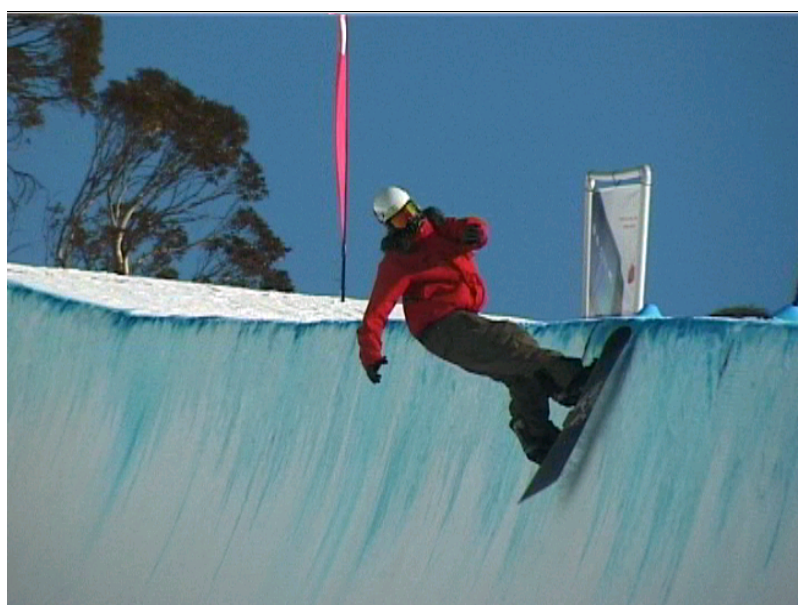

Figure 4. The moment the last part of the snowboard leaves the transition surface of the half-pipe was deemed the beginning of an aerial acrobatic manoeuvre and the beginning of the calculation of air-time ${ }^{11}$. Perisher Blue Australia 2006.

\subsection{Signal processing of accelerometer data}

Large data sets from entire practice sessions had to be reduced to individual runs. Once the capacity to detect individual half-pipe snowboard runs was possible, a signal processing technique was developed to calculate the air-time associated with each individual aerial acrobatic manoeuvre performed during all half-pipe snowboard runs detected. Half-pipe snowboarding generates a very distinct raw tri-axial accelerometer data trace. A raw, unfiltered accelerometer data trace (both forward/back and up/down acceleration axes) displaying elevated accelerations throughout aerial acrobatic manoeuvres can be seen in (Figure 5). Both run detection and air-time calculation was possible because of the rapid increases and decreases in acceleration in both the up/down and forward/back accelerometer axes respectively. These rapid changes in acceleration occur when athletes progresses up, out of and eventually re-enter the vertical half-pipe transitions during the performance of aerial acrobatic manoeuvres.

\subsection{Half-pipe snowboarding run detection}

By utilising a sliding Fast Fourier Transform (FFT) window and subsequent power analysis, average power levels were used to detect individual runs. Power levels are raised during half-pipe snowboarding runs as a result of the substantial increases or decreases in acceleration levels throughout the performance of aerial acrobatic manoeuvres. Analysing entire runs for elevated levels of acceleration allowed run locations to be identified and run windows to be extracted. Evaluating each FFT window for frequencies ranging from $0.25 \mathrm{~Hz}-0.85 \mathrm{~Hz}$ (as aerial acrobatic manoeuvres occur relatively rhythmically with a period of 1.2 - 4 seconds), provided a threshold based algorithm the capacity to then 
identify runs within the trace. The threshold for power was set relatively low to ensure no events were missed. Although the technique had the potential to generate false positives the secondary processing technique (which searches for aerial acrobatic manoeuvres housed within each identified run) limited possibility of calculating air-times in false positive runs.

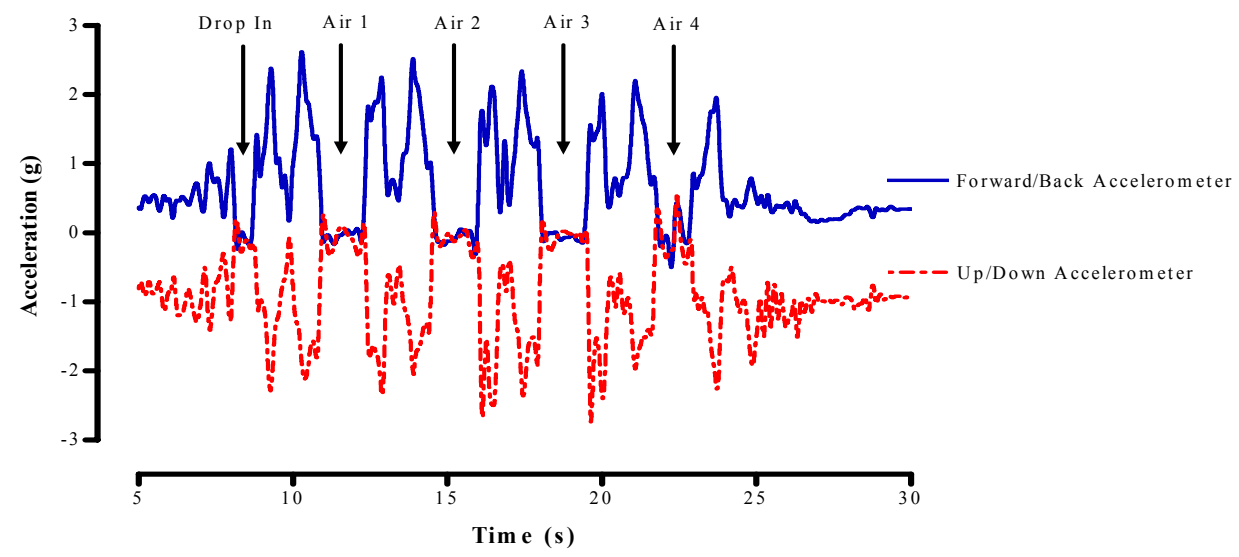

Figure 5. A raw, unfiltered accelerometer trace associated with a half-pipe snowboarding run.

\subsection{Calculation of aerial acrobatic air-time - first pass}

The airtime algorithm was developed using a two pass method. The first pass was comprised of a three state (maximum, minimum and transition) changeover system passed over a complete run accelerometer trace. This transformed the raw accelerometer data within detected run windows into quantised values of 1's 0 's and -1's for high, transition and low states respectively (Figure 6). The high and low threshold values were determined by analysis of 92 aerial acrobatic manoeuvres sampled by this study. The technique then re-analysed the data on an airtime by airtime basis using a much smaller window which further improved accuracy. The high state threshold ratio (TH) was experimentally determined to be 0.25 . The low state threshold (TL) was determined to be 0.3 . Raw accelerometer data transformed into the high state quantised value of 1 gives a crude approximation of when the boarder was in the air and thus the beginning of an airtime. Raw accelerometer data transformed into the low state quantised value of -1 gives an approximation of when the athlete has landed back onto the half-pipe transition and is moving into the flat middle section of the half-pipe. A transition state may include any values between these two threshold values and this procedure essentially eliminates a large proportion of noise and movement not associated with the air-time of aerial acrobatic manoeuvres (Table 1).
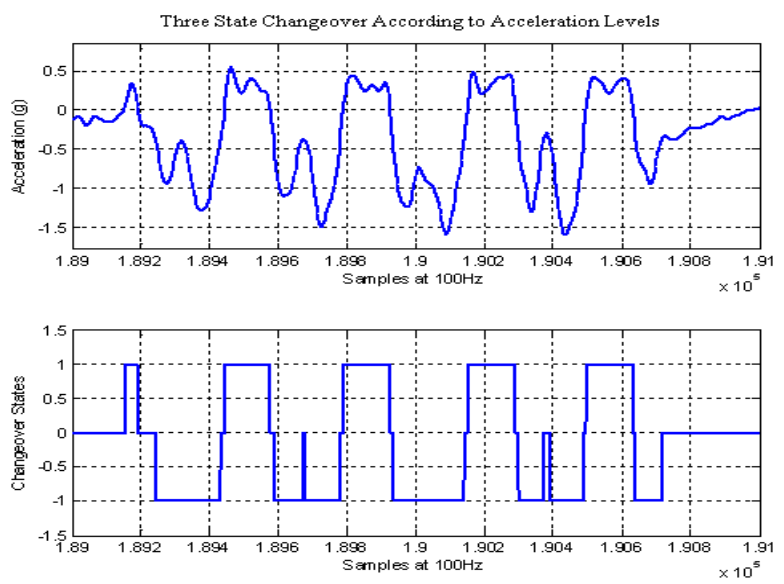

Figure 6. The first pass three state changeover system transforming raw accelerometer data (up/down axis) into the tri-state representation. 
Table 1. The first pass three state changeover system displaying the threshold level associated binary value, and state equation.

\begin{tabular}{|c|c|c|}
\hline Threshold & Binary Value & Equation \\
\hline 0.25 & 1 & High $=$ Data $\geq(\max ($ data $)+$ mean $($ data $)) \cdot \mathrm{T}_{\mathrm{H}}$ \\
\hline $0.25-0.3$ & 0 & Transition $=\mathrm{Low} \leq \mathrm{Data} \leq \mathrm{High}$ \\
\hline 0.3 & -1 & Low $=$ Data $\leq(\min ($ data $)-$ mean $($ data $)) \cdot \mathrm{T}_{\mathrm{L}}$ \\
\hline
\end{tabular}

\subsection{Dynamic movement and potential for error}

Half-pipe snowboard athletes are rarely static when performing aerial acrobatic manoeuvres or when they are moving between the opposite transitions of the half-pipe. In terms of the performance of an aerial acrobatic manoeuvre and the subsequent calculation of air-time, this continual movement can generate a degree of erogenous readings within an accelerometer trace throughout this period where the trace is expected to remain above the quantised level of 1 (Figure 7). Movement artefact arises from rapid movements athletes often make whilst performing aerial acrobatic manoeuvres and are associated with the terms of 'pump', 'tweak' or 'bone' to describe this specific motion. Half-pipe athletes do this for a variety of reasons including maintaining balance during an aerial acrobatic manoeuvre that has been performed incorrectly and often to incorporate an extra component of style and individual flair into their performance. Additionally, athletes regularly undertake similar pumping movements across the flat component of the half-pipe to generate speed prior to progressing up the next half-pipe transition into an aerial acrobatic manoeuvre.

\subsection{Calculation of aerial acrobatic air-time - second pass}

These artefacts caused rapid increases and decreases in acceleration readings (termed spikes) resultant of the sensitivity of tri-axial accelerometers and would subsequently cause the first pass state changeover system (when used alone) to generate incorrect air-time calculations. As a result of this sport specific phenomenon, a second pass technique focused on the possible durations of aerial acrobatic manoeuvres was required. In this second pass, air-times are considered to start (from the quantised values 0 - 1) and air-times finish (from the quantised values 1 - 0 ). All components of a raw accelerometer data trace that meet these criteria are saved into an array (called 'might') whereby the 'might' array is then analysed for air-times that meet a specific duration. The duration of any component that meets the 'might' array criteria must therefore be of a duration between $0.8-2.2$ seconds (a range that would cover most aerial acrobatic air-times completed by half-pipe snowboard athletes) to be considered a valid aerial acrobatic manoeuvre (Figure 8). In the example of a typical run having undergone a state changeover (Figure 6), it is possible to see a number of erroneous spikes occurring between airtimes that are actually caused by the athlete generating speed along the flat component of the half-pipe prior to progressing up the next transition and into an aerial acrobatic manoeuvre. Using the three state changeover system alone would categorise these spikes as an aerial acrobatic manoeuvre and attempt to calculate the associated air-time. The second pass technique (Figure 8) however, eliminated the possibility of these spikes being identified as an air time due to the analysis of the duration of such spikes. 


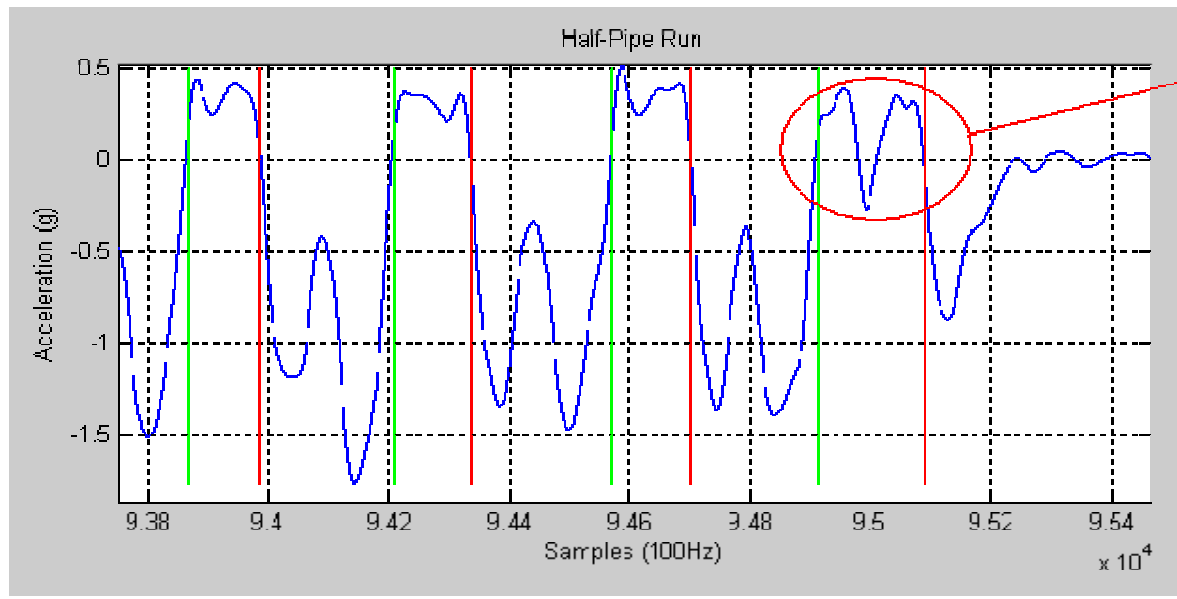

PapidDecrease in

Upwarjs

Acceleration due to

Pumping. (This data

set $s$ f Iterec as the

effect in raw data is

Gveา more profound)

Figure 7. Movement artefact in accelerometer data trace (up/down axis) during an aerial manoeuvre. This is caused when an athlete 'pumps', 'tweaks' or 'bones' the snowboard to maintain balance or show additional individual flair.

(A)

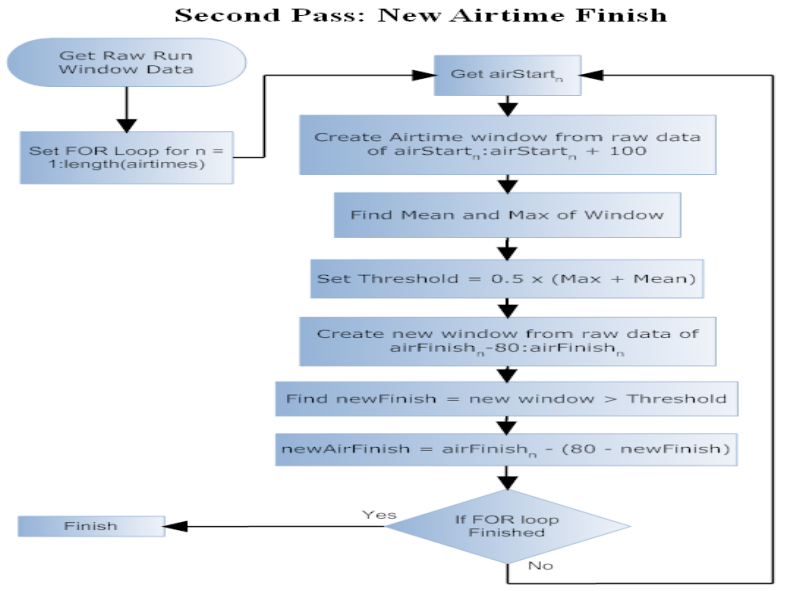

(B)

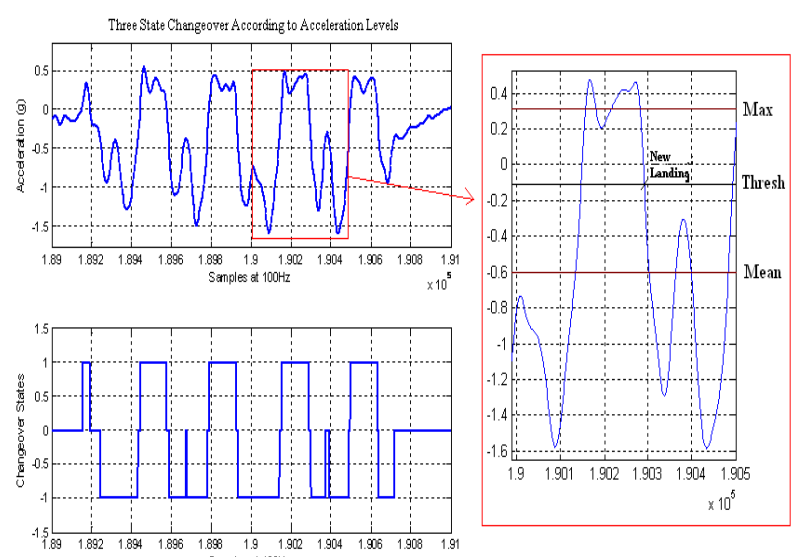

Figure 8. (A) Flow diagram of the second pass algorithm and the second pass air-time window extraction and resultant effect on (B) sample data.

\section{RESULTS}

\subsection{Validation}

This study allowed a criterion-referenced validation of the air-times (mean $1.28 \mathrm{~s} \pm \mathrm{SD} 0.13 \mathrm{~s}$ ) associated with 92 aerial acrobatic manoeuvres calculated by the accelerometer based practical method presented in this paper (all results are presented as value \pm or $\times / \div 95 \%$ confidence limits). There was a very large ${ }^{11}$ correlation $(\mathrm{Y}=0.7477 \cdot$ practical measure of air-time $+0.3021 ; \mathrm{r}=0.78 \pm 0.08 ; \mathrm{p}$ value $<0.0001 ; \mathrm{SEE}=0.08 \times 1 \div 1.16 ; \mathrm{n}=92$ ) between the criterion (video based analysis, y-axis) and practical (accelerometer based signal processing technique, $x$-axis) methods (Figure 9). The mean bias between the criterion and practical measures of air-time was $-0.03 \pm 0.02 \mathrm{~s}$ and the $95 \%$ confidence limits related to the mean bias were $-0.01 \mathrm{~s}$ and $-0.05 \mathrm{~s}$ (Figure 10). There was however, indication of a small degree of non-uniformity in the residuals (heteroscedasticity) shown for example by the bias of $0.05 \mathrm{~s} \pm 0.04 \mathrm{~s}$ at the low end of the air-time scale (1.00s of air-time) and the bias of $-0.10 \mathrm{~s} \pm 0.04 \mathrm{~s}$ at the high end of the air-time scale (1.60s of air-time). Approximately 
$95 \%(94.57 \%)$ of the practical measures lie within $\pm 0.15 \mathrm{~s}$ of the criterion measure. The two pass signal processing technique presented within this paper was able to detect 100 percent of the aerial acrobatic manoeuvres performed.

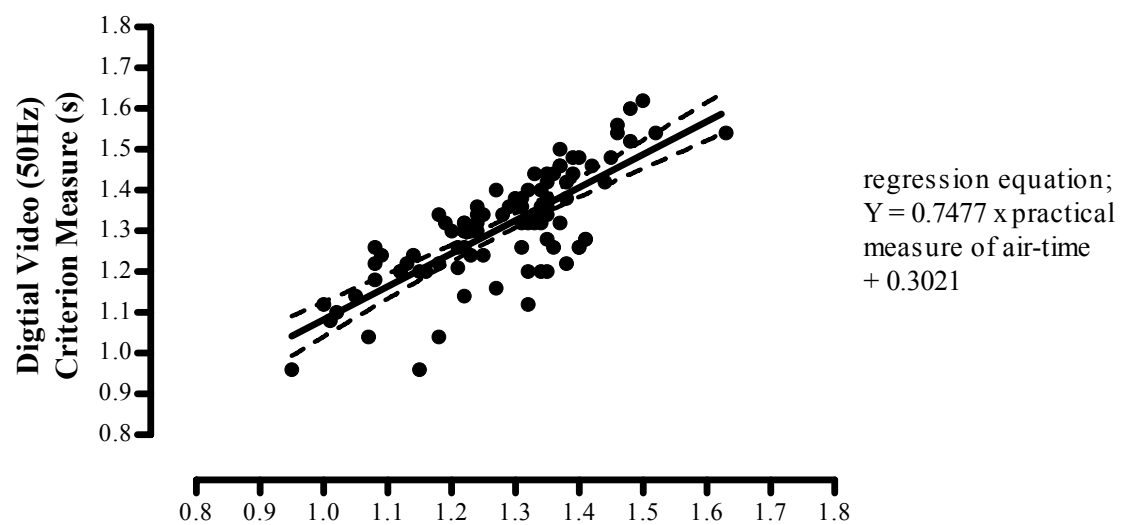

Accelerometer Based Practical Measure (s)

Figure 9. The correlation $(r=0.78 \pm 0.08 ; \mathrm{p}$ value $<0.0001 ; \mathrm{SEE}=0.08 \times / \div 1.16 ; \mathrm{n}=92)$ between the criterion method (video analysis) and the practical method (two pass signal processing technique) for air-time calculation.

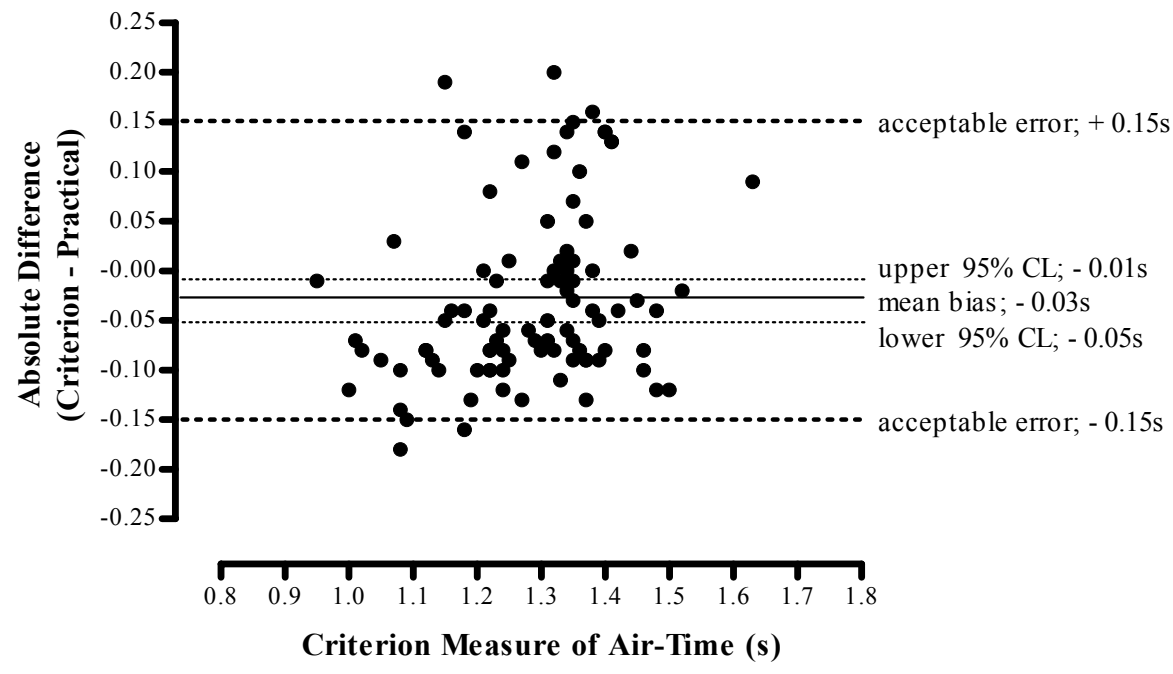

Figure 10. The absolute difference between the criterion and practical methods (criterion - practical) for air-time calculation (mean bias $=-0.03 \pm 0.02 \mathrm{~s}$ ). Approximately $95 \%$ (94.57\%) of the data points lie within $\pm 0.15 \mathrm{~s}$ of the criterion method.

\section{DISCUSSION}

\subsection{Accuracy and reliability of signal processing technique to calculate air-time}

The validity associated with calculation of air-time using the accelerometer based practical method was tested against the video based criterion method. This paper has shown that there is a very large ${ }^{12}$ correlation between the air-time (associated with aerial acrobatic manoeuvres performed during half-pipe snowboarding) calculated by the accelerometer based practical method presented in this paper and the video based criterion method $(\mathrm{r}=0.78 \pm 0.08 ; \mathrm{p}$ value $<0.001$; $\mathrm{SEE}=0.08 \times / \div 1.16$; mean bias $=-0.03 \pm 0.02 \mathrm{~s} ; \mathrm{n}=92$ ). Although the author has previously showed a larger, almost perfect $^{12}$ correlation $(\mathrm{r}=0.92, \mathrm{SEE}=0.05, \text { mean bias }=0.01 \mathrm{~s}, \mathrm{n}=102 \text { aerial acrobatic manoeuvres })^{1,8}$ between an accelerometer based practical method (a threshold based evaluation of raw accelerometer data running averages) and the video based criterion method, the practical method presented in this paper was considered to be more reliable and a 
superior method. This was because the practical method presented in this paper was able to detect 100 percent of the aerial acrobatic manoeuvres performed throughout the study as opposed to the 73 percent detection previously shown by the author ${ }^{1,8}$. False positive air-time calculation would have an overwhelming detrimental effect on any automated feedback provided within elite-level coaching or judging environments. Subsequently, the capacity of the technique to reliably detect air-times with the absence of false positives was considered to be an extremely important factor for the successful integration of this concept into elite-level half-pipe snowboard training and competition protocols.

The mean bias $(-0.03 \pm 0.02 \mathrm{~s})$ shows the practical method on average, measures air-time slightly higher than the criterion method (Figure 10). This could potentially be resultant of the criterion method which, although the reference standard, was not perceived to be without its own inherent bias. Furthermore, the criterion method begins the first moment there is no longer contact between the snowboard and the snow and ends the moment any part of the snowboard comes in contact with the snow following an aerial acrobatic manoeuvre. The criterion method therefore has the potential to detect air-time start and end points that could be difficult for an accelerometer based system to indentify. The practical method is vulnerable to inherent noise associated with sensor attachment and relies on accelerometers to detect impact or the absence of it. There is the possibility that certain criterion method air-time start and end points are void of the significant alterations in impact and acceleration levels the practical method relies upon. There was however a degree of nonuniformity in the bias between the criterion and practical methods. The bias inherent in the practical method at low and high ends of the air-time scale (for example 1.00s and 1.6s of air-time) were somewhat larger than the mean (bias $=-0.05$ $\pm 0.04 \mathrm{~s}$ and $0.10 \mathrm{~s} \pm 0.04 \mathrm{~s}$ respectively). At low air-times it is possible for the practical method to measure higher and for high air-times it is possible for the practical method to measure lower than the criterion method. There was however, no significant relationship in the non-uniformity of this bias. It seems the non-uniformity was generated by outliers rather than being a systematic effect of increasing air-time. As such the utilisation of a calibration equation is of no use in correcting the slight non-uniformity within this data sample. Approximately $95 \%(94.57 \%)$ of the practical measures lie within $\pm 0.15 \mathrm{~s}$ of the criterion measure. Although $\pm 0.15 \mathrm{~s}$ of error $(11.7 \%$ error at the average air-time of $1.28 \mathrm{~s})$ was deemed to be acceptable for the purpose of this concept, further research will focus on reducing the mean bias associated with the practical method. Improved accuracy will always be a focus however, the capacity of the practical method to calculate air-time for 100 percent of aerial acrobatic manoeuvres where $94.7 \%$ of measures fell within $\pm 0.15 \mathrm{~s}$ of the criterion will allow successful integration of this concept into half-pipe snowboarding training environments.

\subsection{An optimal ideology}

This paper has presented the rationale, signal processing technique and validation for an accelerometer based system that calculates quantitative information on air-time associated with half-pipe snowboarding. The focus is well positioned within the current shift in performance assessment ideology; moving data collection and analysis away from the laboratory and into the field. Strong emphasis however was placed on the relevance of the information provided by this system. The relevance of the air-time associated with aerial acrobatic manoeuvres executed during elite competition has been previously reported by the author. The knowledge that air-time comprises a large component of an athletes' subjectively judged score provided strong rationale behind the development of signal processing techniques to automatically calculate objective information on air-time. This was not scientific enquiry initiated without reason, but for practical application within an Olympic sporting discipline. This paper has promoted the use of micro-technologies to propel field based performance assessment in half-pipe snowboarding from futuristic vision to functional reality. The lightweight, unobtrusive nature of micro-technologies and their capacity to measure human motion thousands of times per second in multiple axes provides a realistic manner of measuring the natural, unconstricted and unaltered movement patterns of elite athletes performing in high risk sports. The challenge however seems to be using these capabilities to calculate objective information which is accurate, reliable and most importantly, of relevance to the end user.

\subsection{Future directions}

Air-time, whether averaged (AAT) or totalled (TAT) is not the only objective performance variable that impacts on an athlete's subjectively judged competition score. The author has previously shown the average degree of rotation (ADR) associated with a half-pipe snowboard run also contributes to competition scores ${ }^{1,8,11}$. Therefore, a likely extension of this research would be to develop and validate a signal processing technique to characterise variations in aerial acrobatic manoeuvres and calculate the exact degree of rotation (DR). It is proposed the raw data obtained from tri-axial rate 
gyroscopes combined with the practical method of air-time calculation presented in this paper will enable this future direction to become a reality. Although this paper focused upon the development of an automated feedback system for enhancing athletic performance, this concept has potential application within the current subjective judging protocols used in elite half-pipe snowboard competition. Future work will focus on the potential of an automated feedback system to assist elite-level half-pipe snowboard competition judges to minimise subjective bias and promote reliability in competition performance assessment. Furthermore, it is proposed that any sporting discipline that is comprised of an aerial acrobatic component can benefit from this research. The potential applications of the proposed automated feedback system within the sporting disciplines of half-pipe skateboarding and aerial surfing will also be investigated.

\section{CONCLUSION}

Although this research has been action based and involved a progressive partnership ideology with the practice community, the author foresees a challenge for elite half-pipe snowboarding competition in the future. The technological advancements presented in this paper are theorised to enhance athletic performance and assist subjective judging protocols however, such innovation necessitates a balanced approach observant of a sporting discipline's underlying cultural ethos. It is imperative that equilibrium between the focus of scientific research and sporting community expectations be reached for the successful integration of any technological innovation into elite sport.

\section{ACKNOWLEDGEMENTS}

The authors would like to thank Ben Wordsworth and Ben Alexander (Australian national half-pipe snowboard coaches) and the athletes of the Australian Half-pipe Snowboard Team for their overall support and participation in this project. The authors would like to thank Heidi Barbay for research assistance and provision of photographic images during numerous southern hemisphere winter seasons. The authors would also like to thank the Australian Sports Commission Applied Research Centre general and collaborative grant scheme, Olympic Winter Institute of Australia, Griffith University, Perisher Blue Ski Resort, NSW National Parks and Wildlife Service and Catapult Innovations for all funding and in-kind support. Your support is gratefully acknowledged for without it the research would have never been possible.

\section{REFERENCES}

1 Harding, J. W., Toohey, K., Martin, D. T., Mackintosh, C., Lindh, A. M., James, D. A. (2007) Automated Inertial Feedback For Half-Pipe Snowboard Competition And The Community Perception. The Impact of Technology on Sport II, Fuss F. K., Subic A., Ujihashi S. Taylor \& Francis London., 20, $845-850$.

2 Doki, H., Yamada, T., Nagai, C. Horaki, M. (2005) Development of a measurement system for snowboarding turn analysis. The Impact of Technology in Sport, Subic, A., and Ujihashi, S. ASTA, $324-325$.

3 Delorme, S., Tavoularis, S. (2005) Kinematics of the Ankle Joint Complex in Snowboarding. Journal of Applied Biomechanics, 21, $394-403$.

4 Bianchi, L., Petrone, N., Marchiori, M (2004) A dynamometric platform for load data acquisition in snowboarding: design and field analysis. The Engineering of Sport 5, Hubbard, M., Mehta, R. D., Pallis, J.M. ISEA, 2, 187 -193.

5 James, D. A., Davey, N., Rice, T. (2004) An Accelerometer Based Sensor Platform for Insitu Elite Athlete performance Analysis. IEEE Sensors, Vienna.

6 James, D. A. (2006) The Applications of Inertial Sensors in Elite Sports Monitoring. The Engineering of Sport 6, Moritz, E. F., Haake, S. Springer Science + Business Media, LLC., 3, $289-294$.

7 Michahelles, F., Schiele, B. (2005) Sensing and Monitoring professional Skiers. IEEE Pervasive Computing, 4, 40 - 46.

8 Harding, J. W., Mackintosh C., Martin D. T., Rosemond D., Dowlan S., James D. A. (2006) Applications for inertial sensors in elite-level half-pipe snowboarding. Paper given at the $17^{\text {th }}$ Biennial Congress of the Australian Institute of Physics, Brisbane, 3 8 December, abstract number 484.

9 Mini Max, Catapult (2007) Catapult Innovations Melbourne Australia.

10 Matlab high performance numeric computation and visualisation software (2006) Mathworks USA, release 14.

11 Harding, J. W., Mackintosh C., Martin D. T., Rosemond D., Dowlan S., James D. A. (2005) Key Performance Variable Detection and Validation of an Accelerometer-Based Algorithm to Calculate Air-Time During Half-pipe Snowboarding. Paper given at NESC Applied Physiology Conference, Canberra, 27-28 October.

12 Hopkins, W. G (2000) Analysis of validity by linear regression (Excel spreadsheet). In: A new view of statistics. sportsci.org: Internet Society for Sport Science, sportsci.org/resource/stats/xvalid.xls. viewed on 2 November 2007. 